\section{PSICOLOGIA IBEROAMERICANA}

\section{Psicología lberoamericana}

ISSN: 1405-0943

psicología.iberoamericana@uia.mx

Universidad Iberoamericana, Ciudad de

México

México

Enríquez Jiménez, Francisco Javier; Domínguez Espinosa, Alejandra del Carmen Influencia de la Deseabilidad Social (DS) en Reportes de Capacitación

Psicología Iberoamericana, vol. 18, núm. 1, enero-junio, 2010, pp. 69-79

Universidad Iberoamericana, Ciudad de México

Distrito Federal, México

Disponible en: http://www.redalyc.org/articulo.oa?id=133915936008

Cómo citar el artículo

Número completo

- Más información del artículo

- Página de la revista en redalyc.org

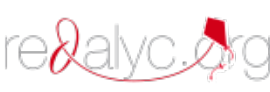

Sistema de Información Científica

Red de Revistas Científicas de América Latina, el Caribe, España y Portugal

Proyecto académico sin fines de lucro, desarrollado bajo la iniciativa de acceso abierto 


\title{
Influencia de la Deseabilidad Social (DS) en Reportes de Capacitación
}

\author{
Social Desirability Responding Influence In Training Report Evaluations
}

\author{
Francisco Javier Enríquez Jiménez * \\ Alejandra del Carmen Domínguez Espinosa** \\ Petróleos Mexicanos, Universidad Nacional Autónoma de México, CD. de México* \\ Universidad lberoamericana, CD. de MÉxICO**
}

\section{RESUMEN}

El propósito del presente estudio fue obtener evidencia del efecto de las instrucciones Socialmente Deseables en los resultados de los Cuestionarios que evalúan la Capacitación (CEC). El CEC mostró un índice adecuado de consistencia interna $(\alpha=.92)$, una validez de factorial de 71.08\%. Como medición de DS se utilizó la escala de Marlowe y Crowne (MCSDS) con un $\alpha=.778$, en sus dimensiones de atribución y negación. Se consideraron instrucciones mediante la inducción de respuestas socialmente deseables $v s$. estándar e instructores internos $v s$. externos. Las dos escalas mostraron asociaciones bajas pero significativas $(r=.187, p<.01)$. La correlación mayor se encontró en la interacción entre la instrucción estándar y el instructor interno, principalmente en la dimensión de atribución. A pesar de tratarse de una evaluación de un tercero, hay evidencia de que las personas puedan estar sesgadas en sus juicios y presentar distorsiones en las respuestas atribuibles a la DS.

Descriptores: Deseabilidad social, instrucciones socialmente deseables, evaluación de la capacitación, psicología organizacional, Marlowe-Crowne.

\section{ABSTRACT}

The purpose of this study was to obtain evidence of the effect that the Socially Desirable instructions have in the results of the CEC (for its initials in Spanish for Training Evaluation Questionnaire). A preliminary psychometrics analysis of CEC revealed an index of internal consistency of $\alpha=.92$, and factorial validity that explains $71.08 \%$ of the variance. To measure the DS the Marlowe-Crowne Scale (MCSDS) was administrated which also observed an internal consistency of $\alpha=.778$, based in their two theoretical dimensions: attribution and denial. The procedure considered two types of instructions (socially desirable induced responses vs. Standard) and two types of instructors (internal vs. external). Correlation analysis showed low but significant associations $(r=.187, p<.01)$, between both scales. The major index of correlation found was among the interaction of the standard instruction and the internal instructor mainly with the dimension of attribution. Despite of being an evaluation of a third person, there is evidence that implies that people can be biased in their judgments and show distortions in the answers conferred to social desirability.

Key words: Social desirability, socially desirable instructions, training evaluation, organizational psychology, Marlowe-Crowne.

\footnotetext{
* Para correspondencia con el Mtro. Francisco Javier Enríquez Jiménez, dirigirse a Petróleos Mexicanos, Av. Marina Nacional No. 329 "C3", Col. Petróleos Mexicanos, Delegación Miguel Hidalgo, C. P. 11311, teléfono: 19442500. Ext. 56 756, correo electrónico: francisco.enriquez30@yahoo.com Para correspondencia con la Dra. Alejandra Domínguez Espinosa, dirigirse a Prolongación Paseo de la Reforma No. 880, Edif. "J", 2do. Piso, Col. Lomas de Santa Fe, teléfono 5950-40-00. Ext. 4876, correo electrónico: alejandra.dominguez@uia.mx.
} 


\section{INTRODUCCIÓN}

Dentro del área de acción de los psicólogos organizacionales se encuentra la planeación y organización de las actividades de capacitación de los empleados. Los cursos de capacitación incorporan, dentro de sus sistemas de control de calidad, evaluaciones acerca de las actividades y de las habilidades de los instructores, con el objetivo principal de comprobar si la capacitación ha sido eficaz de acuerdo con los propósitos planteados. Sin embargo, los instrumentos con los cuales se realizan dichas evaluaciones no son sujetos de escrutinio ni se comprueban sus cualidades psicométricas, por lo que, en ocasiones, las estimaciones que se desprenden de ellos pueden proporcionar información ineficaz o incierta.

Gran parte de la literatura científica ha proporcionado evidencia acerca de las principales fuentes de varianza de error que muestran las herramientas de auto reportes, tales como la aquiescencia, las respuestas extremas y en particular la deseabilidad social (DS) (Anastasi \& Urbina, 1998). Esta última fuente de invalidación hace referencia a cuando las personas tienden a mostrarse a sí mismas de acuerdo a las normas sociales establecidas de una manera exagerada o poco habitual. La DS implica sobredimensionar las actitudes y comportamientos socialmente deseables y reducir los comportamientos y actitudes socialmente desaprobados o menos deseables (Collazo, 2005; Coq \& Asián, 2002; Crowne \& Marlowe, 1960; Ellington, Sackett, \& Hough, 1999; González \& Iruarrizaga, 2005; Paulhus, 1984). La DS se evidencia cuando el sujeto escoge una respuesta deseable pero poco frecuente en contraposición a la conducta más común pero menos deseable, o bien cuando se elige el reactivo por la conveniencia social de la respuesta (Braun, Jackson, \& Wiley, 2002; Kerlinger \& Howard, 2002; Nunnally \& Bernstein, 1995).

Una de las técnicas o procedimientos que se emplean con más frecuencia para evidenciar la contaminación de una medición por DS es utilizar una escala explicita de DS en conjunto con la escala de interés y verificar si existe una asociación significativa y moderada entre ellas; de ser así, se asume la presencia de DS (Havercamp \& Reiss, 2003; Kellar, Treadwell, Kumar, \& Leach, 2002; Kolotkin \& Crosby, 2002; Mikolajczak, Luminet, Leroy, \& Roy, 2007; Rahim, 1982, 1983,
1988; Robertson \& Milner, 1983; Schludermann \& Schludermann, 2007; Wichstrom, 1995; Williams et al., 1988)

Principalmente, la DS es una fuente de invalidez cuando de manera directa se involucra la autoimagen de la persona evaluada, es decir, cuando se requiere que las personas digan algo acerca de ellas mismas. Se supone que una técnica más general para evitar este efecto es parafrasear los reactivos en tercera persona, lo que permite que disminuya el involucramiento, con lo cual la situación se hace menos amenazante para quien responde y, por ende, proporciona más probabilidades de que conteste sin DS si se utiliza el anonimato (Paulhus \& Reid, 1991; Stöber, Dette, \& Musch, 2002; Van de Vijver, 1997, 1998).

No obstante, cuando el sujeto se encuentra en una situación de demanda social y no es posible el anonimato, la DS se presenta, incluso aunque la evaluación sea de terceros, ya que al hablar mal o bien acerca de otros también damos una impresión de nosotros hacia los demás (Gordon \& Petty, 1971; Konstabel, Aavik, \& Allik, 2006).

\section{Evaluación de la capacitación y sus beneficios}

La capacitación está orientada a satisfacer las necesidades de las organizaciones de incorporar conocimientos, habilidades y actitudes en sus miembros, como parte de su natural proceso de cambio, crecimiento y adaptación a nuevas circunstancias internas y externas. Sin embargo, la medición de los resultados de las acciones de capacitación en el marco de las organizaciones es probablemente el aspecto menos desarrollado de los que componen esta disciplina. Ello se debe a lo difícil, pero posible, que es medir los resultados de la capacitación, en razón de la subjetividad implícita de lo que se va a medir, debido al alto impacto de diversas variables sobre el comportamiento humano, y por último por la magnitud del esfuerzo que significa una medición en materia de tiempo, recursos, etcétera (Blake, 2000).

Cuando se habla de capacitación se hace referencia a un proceso mediante el cual se trata de obtener determinados beneficios que justifican su existencia, por ejemplo: menos accidentes de trabajo, menor rotación, incremento de las aptitudes, mayor productividad o 
eficiencia organizacional, etcétera (véase a Chiavenato, 2000; Grados, 1998).

Sin evaluación, es muy difícil demostrar que la capacitación fue la razón de las mejoras. De la formación de los recursos humanos se puede garantizar la productividad, así como la preparación de los empleados actuales para futuros puestos de trabajo. Los resultados que se utilizan en la evaluación de programas de formación incluyen la satisfacción que generan los programas de capacitación, el aprendizaje de conocimientos o habilidades, el uso de conocimientos teóricos y prácticos en el trabajo, y en los resultados como en las ventas, la productividad o la prevención de accidentes. Investigaciones previas sobre el efecto de los programas de la capacitación en los Recursos Humanos han comprobado los beneficios tangibles e intangibles de la capacitación y el retorno de la inversión (véase Bryant \& Terborg, 2008; Fraser, 2007; Kumpikaite, 2007).

El objetivo de la evaluación es investigar actitudes, aprendizaje, comportamientos o bien el nivel de conocimiento o información que tienen los capacitados (Bentley, 1993; Grados, 2001).

Para que la capacitación resulte eficaz influyen diversos factores, tales como la selección del personal, los procedimientos de situación de empleados en sus puestos, las diferencias individuales en aptitudes, temperamento y rapidez de aprendizaje, etcétera (McGehee \& Thayer, 1986).

La mayoría de los estudios sobre los programas de capacitación concentran su atención en la importancia de la evaluación en forma sistemática y en la forma en que los resultados de ella han ayudado a los directores, los empleados y a los profesionales de los recursos humanos a tomar decisiones sobre la mejora de los programas y los métodos de capacitación, de los costosbeneficios y del costo-eficacia, elementos de mucha utilidad para el análisis de la rentabilidad de la capacitación (Dewine, 1987).

Rodríguez (1993) afirma que los instrumentos de evaluación deben ser diferentes de acuerdo con la diversa naturaleza de los eventos, es decir, pueden contener una serie de reactivos que llevan al participante a emplear la información, las habilidades y las actitudes aprendidas para la solución de determinados problemas. Por esta razón, un cuestionario que evalué la eficacia de la capacitación estará bien elaborado si sus re- activos corresponden a los objetivos y constituyen una buena muestra de los contenidos que se manejaron durante el curso.

Guzmán \& Sánchez Escobedo (2006), realizaron un estudio con el propósito obtener evidencia acerca del efecto de un programa de capacitación para profesores de una institución de educación superior del sureste de México: la Universidad del Mayab (Unimayab). Los autores concluyen que la capacitación docente puede mejorar el desarrollo de habilidades de pensamiento crítico en los estudiantes, pero aún se discuten las limitaciones del estudio y las implicaciones para el entrenamiento de profesores a nivel licenciatura.

La valoración del adiestramiento no es nada sencillo, ya que se requiere determinar los cambios en las habilidades, conocimientos y actitudes de los empleados que deben tener lugar cuando se ven sometidos a un proceso llamado "adiestramiento". Asimismo, determinar la forma en que esas habilidades, conocimientos y actitudes contribuyen a la consecución de los objetivos de la organización tampoco es tarea fácil. Para resolver estas cuestiones, deben crearse medidas confiables y válidas a fin de determinar si el entrenamiento hace en verdad lo que se espera que haga (Smith \& Wakeley, 1988). La evaluación de la capacitación es un proceso que debe concebirse en términos de medición. Por ello, debe centrarse en recabar información de varios escenarios laborales en los que se hayan implantado programas de capacitación y en donde los objetivos inicialmente establecidos se hayan convertido en procesos realizados y hayan sido aplicados por los empleados capacitados, dentro de su contexto de trabajo (Grados, 1998).

\section{Deseabilidad social}

El punto de partida del constructo de la deseabilidad social es el supuesto de que algunas de las alternativas de respuesta ante ciertos reactivos de personalidad son socialmente más convenientes o deseables que otras, por lo que algunos sujetos pueden tender a elegirlas con independencia de cuál sea su nivel auténtico en el rasgo que el reactivo pretende medir (Ferrando \& Chico, 2000). La mentira, el engaño o la ocultación, la deformación y tergiversación de la información son conductas consustanciales al ser humano, que persiguen diversos fines en el proceso de interacción so- 
cial, como ofrecer una impresión positiva a los demás, autobeneficiarse y/o beneficiar a otros, evitar un castigo potencial o simplemente mantener una buena interacción social procurando no herir de manera innecesaria los sentimientos de los demás (Martínez Selva, 2005). Por su parte, Homanns (1974 en Harry, 1984) sugiere que la DS tiene su origen en la necesidad de la aprobación social, la cual es concebida como un factor de motivación importante del comportamiento humano y que existe una disposición general de parte de individuos para buscar la evaluación favorable de otros.

Anastasi y Urbina (1998), sostienen que hay personas que se sienten motivadas a distorsionar sus respuestas para "quedar bien" ante los demás, es decir, elegir respuestas que generen una impresión favorable, como cuando solicitan empleo o intentan ingresar a una institución educativa. Sin embargo, también hay personas motivadas a distorsionar sus respuestas para "quedar mal", es decir, tratan de aparentar un trastorno psicológico mayor al que presentan, por ejemplo, en la evaluación de quienes están sometidos a un juicio por haber cometido un delito. En ambos casos, la distorsión implica que las mediciones que se hacen no son confiables y pueden llevar al evaluador a conclusiones erróneas o diagnósticos mal definidos, de tal suerte que se hace necesario poder identificar a aquellos sujetos que de manera intencional distorsionan sus respuestas.

La deseabilidad social consta de dos dimensiones, que han sido denominadas manejo de impresiones (impression management) y autoengaño (self-deception). El manejo de impresiones indica la tendencia a adaptar de manera intencional la imagen pública de uno mismo con objeto de obtener una visión favorable por parte de los demás. El autoengaño, por el contrario, se refiere a la tendencia no intencionada de describirse a uno mismo de un modo favorable y que se manifiesta en autodescripciones sesgadas positivamente pero en las que se cree a pie juntillas. En este sentido, el manejo de impresiones es una manipulación voluntaria de la propia imagen para que los demás lo perciban a uno de modo positivo, mientras que el autoengaño no es una manipulación deliberada aunque pueda conducir a distorsiones en la percepción que los otros tienen de uno (Salgado, 2005).
Al igual que con cualquier medida de lápiz y papel de las características y comportamientos humanos, un problema relacionado con los inventarios de personalidad se vincula con su validez. Muchos de los reactivos de los inventarios requieren que quienes responden admitan cosas que podrían desear no admitir, sino, por el contrario, presentarse de la manera más favorable. La veracidad cuando se debe responder puede ser un problema grave y es probable que los individuos no estén dispuestos a decir la verdad (Aiken, 2003).

Muchos investigadores han buscado la forma de medirla y, si es necesario, controlarla para asociarla a las distorsiones en los auto reportes. Una importante alternativa en esta dirección ha sido el desarrollo de escalas para medir las diferencias individuales en la respuesta socialmente deseable. A través de los años, muchas de estas escalas han sido desarrolladas y aplicadas ampliamente en diversos campos de investigación básica, pero sus usos en la práctica han sido cuestionados por la forma en que se debe evaluar y controlar la deseabilidad social en los inventarios auto descriptivos.

\section{La escala de deseabilidad social de Marlowe- Crowne (MCSDS)}

Hace unos 40 años, Crowne y Marlowe (1960) desarrollaron una nueva escala para medir la deseabilidad social debido a que los 39 ítems de la escala de Edwards habían sido construidos con los ítems del Inventario Multifásico de Personalidad de Minnesota (MMPI) y, por lo tanto, muchos de ellos tenían contenido psicopatológico, por lo que la principal crítica de Crowne y Marlowe era que la escala de Edwards confunde la DS con psicopatología. De esta manera decidieron elaborar una escala totalmente nueva con reactivos que incluyeran un mínimo de contenido patológico. Primero, seleccionaron ítems de los cuestionarios de personalidad que describían comportamientos considerados socialmente deseables, pero improbables en ocurrencia o socialmente indeseables pero que se manifiestan con frecuencia, sin hacer caso de ítems con implicaciones psicopatológicas. Este conjunto de los ítems parecían tener buenas características psicométricas (consistencia interna $=0.88$, test-retest $r=0.89$ ), por lo que los autores llegaron a una nueva escala, la escala de Deseabilidad social de Marlowe y Crowne, 
conocida hoy en día como SDS, por sus siglas en inglés (O’Grady, 1988).

La MCSDS es una de las distintas escalas que se aplican para evaluar diferencias individuales en deseabilidad social. En la actualidad, la versión de la MCSDS está constituida por 33 ítems que se contestan por elección forzada entre Verdadero (V) y Falso (F). A mayor puntaje corresponde mayor deseabilidad social. 18 de los ítems son denominados como atribución. Los ítems de atribución se refieren a la aprobación social de conductas no comunes. El resto de los ítems son considerados de negación y se refieren a conductas socialmente impropias pero comunes (Ramanaiah \& Martin, 1980). Esta herramienta ha sido utilizada para evaluar los sesgos de respuestas en un sentido socialmente deseable en diversas áreas de investigación en Medicina y Psicología (Beretvas, Meyer, \& Leite, 2002; Ramanaiah \& Martin, 1980).

A pesar de que el MCSDS ha sido muy usado por su confiabilidad y validez, existen algunos puntos de vista que reclaman que los ítems de la escala necesitan ser actualizados. Stöber (2001), sostiene que un cierto contenido de la escala del Marlowe-Crowne puede ser anticuada. Se cree que los ítems como "ha habido ocasiones en las que he tenido ganas de revelarme en contra de personas con autoridad aun cuando sabía que tenían la razón" y "en ocasiones he insistido arduamente para que las cosas sean como yo quiero", podrían reflejar estándares sociales de finales de la década de 1950, cuando la escala de Marlowe-Crowne fue construida, y la cuestión es que si aún reflejan los estándares particularmente de los estudiantes universitarios de hoy.

Lo cierto es que el MCSDS es una de la escalas para medir y controlar el sesgo en los estilos de respuestas en los autor reportes, pero desde su aparición se han propuesto y adoptado diversas versiones cortas en el campo de la psicología y la medicina, en una gran variedad de poblaciones, sin pérdida significativa de confiabilidad (Avery \& Gary, 1985; Barger, 2002; Loo \& Thorpe, 2000; Ray, 1984; Reynolds, 1982; Seol, 2007; Strahan \& Gerbasi, 1972; Zook II \& Sipps, 1985).

En la actualidad no hay evidencias de distorsión de las respuestas de quienes contestan a los cuestionarios de evaluación de la capacitación, aun cuando se plantea que las decisiones derivadas de ellas tienen im- portantes implicaciones para las personas que lo hacen (vr. gr., promociones a puestos superiores, asignación de nuevas responsabilidades o cambio de funciones y establecimiento de nuevos programas de capacitación). Además, las evaluaciones de capacitación pueden ser afectadas por DS debido a que, aunque se evalúa a un tercero, siempre existe la percepción que se está evaluando al propio empleado, por lo que en el presente estudio se fijó como objetivo probar si un instrumento de capacitación presentaba asociación significativa con una medida criterio de DS y si esto era indicativo de que, a pesar de evaluar a un tercero, la expresión de la propia opinión también asume un involucramiento de la imagen personal ante una situación de demanda social (ambiente laboral).

\section{MÉTODO}

\section{Participantes}

Los participantes de la presente investigación fueron empleados de una empresa paraestatal con sede en ciudad de México, que asistieron a un curso de capacitación de tipo administrativo, técnico, informático, de seguridad física o de desarrollo humano (20 horas mínimo y 40 horas máximo). El tamaño de la muestra fue de 298 participantes, 177 mujeres, 118 hombres (3 no proporcionaron sus datos), cuyos integrantes fueron seleccionados de manera no probabilística intencional. Los sujetos fueron repartidos aleatoriamente entre los dos métodos de recogida de información que se utilizaron. En el caso del primer método de recogida de datos (Instrucción-Evaluación favorable inducida), se utilizaron 140 sujetos, 81 mujeres y 59 hombres, con una media de edad de 42 años y una desviación típica de 10, además de un rango de 21 a 66 años. El segundo grupo (Instrucción-Estándar), estuvo formado por 155 sujetos, 96 mujeres y 59 hombres con una edad media de 44 años y una desviación típica de 10.93, un rango de 18 a 72 años. La media de edad de la muestra total fue de 43 años y una desviación típica de 10.76, rango de 18 a 72 años. En cuanto a la situación laboral, la mayoría de los trabajadores eran de base (definitivos); 93.73\% sindicalizados y sólo $6.27 \%$ de confianza. En el formato del CEC, no se contempla recabar los datos de escolaridad ni antigüedad del trabajador. 


\section{Instrumentos}

El Cuestionario para la Evaluación de la Capacitación (CEC), es una escala tipo Likert, compuesta por 21 reactivos, divididos en secciones temáticas: 10 reactivos dirigidos a evaluar el desempeño del instructor; 2 las instalaciones; 3 evalúan los materiales proporcionados en el curso y 6 miden en términos generales el desarrollo de evento. Los reactivos se presentan en una escala de respuesta de 5 puntos en los límites de 1 (Totalmente en desacuerdo) a 5 (Totalmente de acuerdo). El CEC tiene un coeficiente de confiabilidad de .92 y una varianza acumulada de $71.08 \%$. Este cuestionario ha sido utilizado desde hace 30 años y fue desarrollado de manera explícita por el Instituto Mexicano del Petróleo para PEMEX (anexo I).

La Escala de deseabilidad social de Marlowe y Crowne (MCSDS), que evalúa diferencias individuales en deseabilidad social, está constituida por 33 reactivos que se contestan por elección forzada entre Verdadero (V) y Falso (F). Dieciocho de los reactivos son denominados de atribución y 15 son considerados de negación. En el caso de los reactivos 1, 2, 4, 7, 8, 13, $16,17,18,20,21,24,25,26,27,29,31$ y 33 , a cada respuesta $\mathrm{V}$ le corresponde el puntaje $1 \mathrm{y}$ a cada respuesta F el puntaje 0 . El resto de los ítems se puntúa de forma inversa.

\section{Procedimiento}

Cada año, Petróleos Mexicanos programa una serie de cursos de capacitación para el personal de las oficinas del Centro Administrativo de la ciudad de México. En este caso, dicho programa se llevó a cabo de febrero a septiembre de 2009. Se le dio seguimiento a la programación de cada uno de los cursos hasta su conclusión. De acuerdo con el tipo de instructor que hubiere impartido el curso (ya sea interno o externo) y previo al término del curso, se prepararon las instrucciones (inducidas y estándar) para cada profesor, según el caso. De acuerdo al número de participantes en cada grupo, se ordenó al alzar el Cuestionario de Evaluación de la Capacitación (CEC) y el MCSDS y en la parte superior de éstos se incorporó una hoja blanca tamaño carta con las instrucciones que inducían a evaluar favorablemente al instructor y otro juego con las instrucciones tipo estándar, que procuraba mantener la misma proporción de ambos ejemplares para todo el grupo.
Posteriormente se engraparon y, de manera aleatoria, se entregó un juego a cada participante. El lugar de aplicación fue el aula en donde se llevó a cabo el evento, con espacio suficiente para alojar a 20 participantes a la vez. La aplicación de los cuestionarios fue realizado por un prestador de servicio social de la carrera de psicología, instruido previamente para dar las siguientes indicaciones para el llenado de los instrumentos:

a) En cada sesión debía explicar al personal asistente el objetivo del cuestionario.

b) Cada participante debía escribir su número de empleado y el nombre del instructor y el nombre del evento, en las casillas correspondientes.

c) Debía solicitar a cada participante que leyera y siguiera las instrucciones, a fin de asegurarse de que no tuviesen ninguna duda para el llenado de los instrumentos.

Se aseguró que la aplicación de los cuestionarios se realizara de manera individual y se respondiera a todos los reactivos. Una vez contestados las preguntas se procedió a capturar los resultados en la base de datos del programa SPSS versión 17.

\section{Tratamiento estadístico}

A fin de determinar si existe una relación entre los puntajes de las evaluaciones del desempeño de los instructores de cursos de capacitación y los puntajes de la escala del MCSDS, se utilizará la prueba de correlación de Pearson y posteriormente se agruparán, en primera instancia, por tipo de instructor (interno o externo) y por último por tipo de instrucción (evaluación favorable inducida o tipo estándar) para observar si existe alguna variación entre éstas. Se realizó un análisis de frecuencia para conocer la población por edad y sexo.

\section{Resultados}

En la tabla 1 se muestra el promedio de la Escala de Deseabilidad Social de Marlowe-Crowne (MCSDS) y las calificaciones del Cuestionario de Evaluación de la Capacitación (CEC), sus desviaciones estándar, los puntajes de consistencia interna y las intercorrelaciones entre ambas herramientas. Se encontraron correlaciones bajas pero significativas respecto del CEC y MCSDS, lo que indica que en las estimaciones 
Tabla 1. Intercorrelaciones y estadísticos básicos CEC y MCSDS

\begin{tabular}{|l|c|c|c|c|c|c|c|c|}
\hline \multicolumn{9}{|c|}{ Correlaciones } \\
\hline Escala/Cuestionario & Rango & M & DE & a & 1 & a & b & 2 \\
\hline 1. MCSDS & 0 a 33 & 24 & 5.09 & .778 & - & & & \\
\hline a. Atribución & 0 a 18 & 13 & 3.07 & .670 & $.796^{* *}$ & - & & \\
\hline b. Negación & 0 a 15 & 11 & 3.23 & .775 & $.817^{* *}$ & $.301^{* *}$ & - & \\
\hline 2. CEC & 0 a 105 & 93.45 & 12.44 & 0.92 & $.187^{* *}$ & $.178^{* *}$ & $.125^{*}$ & - \\
\hline
\end{tabular}

Nota. $\mathrm{N}=268$. MCSDS $=$ Marlowe-Crowne Social Desirability Scale, CEC $=$ Cuestionario de Evaluación de la Capacitación. $\alpha=$ Cronbach's $\alpha,{ }^{* *} p<.01,{ }^{*} p<.05$

Tabla 2. Intercorrelaciones del CEC y MCSDS segmentada por tipo de instrucción

\begin{tabular}{|l|c|c|c|c|c|c|c|c|}
\hline & \multicolumn{7}{|c|}{ Correlaciones } \\
\hline Instrucción & \multicolumn{7}{|c|}{ Evaluación favorable inducida ${ }^{1}$} & \multicolumn{4}{c|}{ Estándar $^{2}$} \\
\hline Escala/Cuestionario & 1 & $\mathrm{a}$ & $\mathrm{b}$ & 2 & 1 & $\mathrm{a}$ & $\mathrm{B}$ & 2 \\
\hline 1. MCSDS & - & & & & - & & & \\
\hline a. Atribución & $.806^{*}$ & - & & & $.787^{* *}$ & - & & \\
\hline b. Negación & $.814^{*}$ & $.313^{* *}$ & - & & $.821^{* *}$ & $.295^{* *}$ & - & \\
\hline 2. CEC & $.179^{*}$ & - & $.224^{*}$ & - & $.197^{*}$ & $.275^{* *}$ & - & - \\
\hline
\end{tabular}

Nota. Evaluación favorable inducida1 $\mathrm{N}=123$; Evaluación estándar2 $\mathrm{N}=156, \mathrm{MCSDS}=$ Escala de Deseabilidad Social de Marlowe-Crowne, CEC $=$ Cuestionario de Evaluación de la Capacitación. ${ }^{* *} p<.01,{ }^{*} p<.05$.

de las evaluaciones de la capacitación se presenta una proporción de deseabilidad social.

Los puntajes de las evaluaciones de eventos de capacitación tienen una correlación significativamente más alta con la Escala de Deseabilidad Social de Marlowe-Crowne, cuando se administran de forma paralela con la instrucción tipo estándar $(r=.197, p<$ .05), mientras que el CEC y el MCSDS presentan correlaciones significativamente más bajas $(r=.179, p<$ $.05)$, ante las instrucciones que inducen evaluar favorable el evento de capacitación (tabla 2). Se puede observar que personas que presentan comportamientos considerados como socialmente deseables, pero improbables en ocurrencia (atribución), muestra correlaciones bajas pero significativas ante las instrucciones estándar $(r=.275, p<.01)$.
Al contrastar el CEC y MCSDS por instructor interno y por tipo de instrucción, los puntajes muestran una correlación significativamente más alta cuando no se induce evaluar favorable el evento de capacitación $(r=.262, p<.05)$, que cuando se administra con la instrucción que sí induce a evaluarlo de manera favorable (véase tabla 3). Nuevamente se observa que las personas que presentan comportamientos socialmente deseables, pero improbables en ocurrencia (atribución), muestran correlaciones significativas, en esta ocasión medias-bajas, ante las instrucciones estándar $(r=.421$, $p<.01)$.

Las evaluaciones del instructor externo, tanto con instrucciones que inducen favorecer su desempeño y de tipo estándar, no mantienen relación alguna con el MCSDS y sus dos subescalas (tabla 4). 
Tabla 3. Intercorrelaciones del CEC y MCSDS segmentada por tipo de instrucción e instructor interno

\begin{tabular}{|l|c|c|c|c|c|c|c|c|}
\hline & \multicolumn{7}{c|}{ Correlaciones } \\
\hline \multicolumn{1}{|c|}{ Instrucción } & \multicolumn{2}{|c|}{ Evaluación favorable inducida ${ }^{1}$} & \multicolumn{4}{c|}{ Estándar $^{2}$} \\
\hline Escala/Cuestionario & 1 & $a$ & $b$ & 2 & 1 & a & b & 2 \\
\hline 1. MCSDS & - & & & & - & & & \\
\hline a. Atribución & $.749^{* *}$ & - & & & $.752^{* *}$ & - & & \\
\hline b. Negación & $.778^{*}$ & - & - & & $.781^{*}$ & - & - & \\
\hline 2. CEC & - & - & - & - & $.262^{*}$ & $421^{*}$ & - & - \\
\hline
\end{tabular}

Nota. Evaluación favorable inducida ${ }^{1} \mathrm{~N}=67$; Evaluación estándar ${ }^{2} \mathrm{~N}=94, \mathrm{MCSDS}=$ Escala de deseabilidad social de Marlowe-Crowne, CEC $=$ Cuestionario de Evaluación de la Capacitación. ${ }^{* *} p<.01,{ }^{*} p<.05$.

Tabla 4. Intercorrelaciones del CEC y MCSDS segmentada por tipo de instrucción e instructor externo

\begin{tabular}{|l|c|c|c|c|c|c|c|c|}
\hline & \multicolumn{7}{|c|}{ Correlaciones } \\
\hline Instrucción & \multicolumn{6}{|c|}{ Evaluación favorable inducida ${ }^{1}$} & \multicolumn{4}{c|}{ Estándar $^{2}$} \\
\hline Escala/Cuestionario & 1 & $\mathrm{a}$ & $\mathrm{b}$ & 2 & 1 & $\mathrm{a}$ & $\mathrm{b}$ & 2 \\
\hline 1. MCSDS & - & & & & - & & & \\
\hline a. Atribución & $.873^{*}$ & - & & & $.834^{* *}$ & - & & \\
\hline b. Negación & $.867^{* *}$ & $.513^{* *}$ & - & & $.871^{* *}$ & $.454^{* *}$ & - & \\
\hline 2. CEC & - & - & - & - & - & - & - & - \\
\hline
\end{tabular}

Nota. Evaluación favorable inducida1 $\mathrm{N}=56$; Evaluación estándar $2 \mathrm{~N}=62, \mathrm{MCSDS}=$ Escala de deseabilidad social de Marlowe-Crowne, CEC $=$ Cuestionario de Evaluación de la Capacitación. ${ }^{* *} p<.01,{ }^{*} p<.05$.

\section{CONCLUSIONES}

Considerar a la deseabilidad social (DS) como un estilo de respuesta, que potencialmente afecte las evaluaciones de los instructores que imparten cursos de capacitación, no ha sido documentado hasta ahora dentro de la literatura científica. Al contabilizar cuestionarios de evaluación de la capacitación que se vean afectados con respuestas distorsionadas de manera positiva, se corre el riesgo de evaluar de manera favorable un programa de capacitación que no lo merece. Esta distorsión conlleva a seguir programando la intervención de los mismos instructores o de la continuidad de determinado evento, en vez de realizar los ajustes necesarios o cambios sustanciales más pertinentes. Las consecuencias de una detección deficiente de la eficacia de un curso de capacitación pueden reflejarse drásticamente en la incorrecta formación del personal, el óptimo desarrollo de las tareas y operaciones y, por ende, en un obstáculo para el logro de los objetivos organizacionales.

Se observó un efecto contrario con las instrucciones que inducen a evaluar de manera favorable el desempeño de los instructores, es decir, cuando se siguen instrucciones estándar en vez de manipuladas, los participantes muestran mayor relación con los rasgos de aprobación social. No se descarta la presencia de la DS en los métodos de la evaluación de la capacitación, aun cuando la DS se manifiesta de forma notable en los autores reportes que miden actitudes y 
comportamientos socialmente deseables. Los resultados indican que este estilo de respuesta también puede distorsionar las evaluaciones de los cursos de capacitación con instrucciones estándar y dar una buena impresión de una tercera persona (instructores). Por lo tanto, los cuestionarios que evalúan la capacitación

\section{REFERENCIAS}

Aiken, L. R. (2003). Test Psicológicos y Evaluación (Undécima ed.). México: Pearson Prentice Hall.

Anastasi, A., \& Urbina, S. (1998). Test Psicológicos (7a. ed.). México: Prentice Hall.

Avery, Z., \& Gary, J. S. (1985). Cross-Validation of a short form of the Marlowe-Crowne Social Desirability Scale. Journal of Clinical Psychology, 41(2), 236-238.

Barger, S. D. (2002). The Marlowe-Crowne Affair: Short forms, psychometric structure, and social desirability. Journal of Personality Assessment, 79(2), 286-305.

Bentley, T. (1993). Capacitación Empresarial. Colombia: McGraw-Hill.

Beretvas, S. N., Meyer, J. L., \& Leite, W. L. (2002). A reliability generalization study of the MarloweCrowne Social Desirability Scale. Educational and Psychological Measurement, 62(4), 570-589.

Blake, O. (2000). Origen, Detección y Análisis de las Necesidades de Capacitación. Argentina: Macchi.

Braun, H. I., Jackson, D. N., \& Wiley, D. E. (2002). Socially Desirable Responding: The evolution of a construct. En: Psychological and Educational Measurement, 4969.

Bryant, S. E., \& Terborg, J. R. (2008). Impact of peer mentor training on creating and sharing organizational knowledge. Journal of Managerial Issues, 20(1), 11-29.

Collazo, A. A. (2005). Translation of the MarloweCrowne Social Desirability Scale into an equivalent spanish version. Educational and Pshychological Measurement, 65(5), 780-806.

Coq, D., \& Asián, R. (2002). Estudio de la "deseabilidad social" en una investigación mediante encuestas a empresarios andaluces. Metodología de encuestas, 4(2), 211-225. y que presentan respuestas distorsionadas tienden a producir una buena impresión del instructor o bien, del evaluado al hablar bien del instructor, lo que hace suponer que las evaluaciones de cursos de capacitación son susceptibles de distorsión y pueden dar como resultado serios problemas en la validez de los datos.

Crowne, D. P., \& Marlowe, D. (1960). A new scale of social desirability independent of psychopathology. Journal of Consulting Psychology, 24(4), 349-354.

Chiavenato, I. (2000). Administración de Recursos Humanos. Colombia: McGraw-Hill.

Dewine, S. (1987). Evaluation of organizational communication competency. Journal of Applied Communication Reserch, 15(1-2), 113-127.

Ellington, J., Sackett, P., \& Hough, L. (1999). Social desirability corrections in personality measurement: issues of applicant comparison and construct validity. Journal of Applied Psychology, 84 155-166.

Ferrando, P. J., \& Chico, E. (2000). Adaptación y análisis psicométrico de la escala de deseabilidad social de Marlowe y Crowne. Psicothema 12(3), 383-389.

Fraser, J. (2007). Developing a framework to evaluate training programs provided by WHO: The feasibility of incorporating social justice, cultural competency and return on investment. The international journal of learning, 14(9), 103-109.

González, H., \& Iruarrizaga, I. (2005). Evaluación de las distorsiones de respuesta mediante el MMPI-2. Papeles del Psicólogos, Consejo General de Colegios Oficiales de Psicólogo, España, 26(092), 129-137.

Gordon, M. E., \& Petty, M. M. (1971). A note on the effectiveness of research conditions in reducing the magnitude of dissimulation on a self-report criterion. Personnel Psychology, 24(1), 53-61.

Grados, J. A. (1998). La Capacitación. México: Trillas.

Grados, J. A. (2001). Capacitación y Desarrollo de Personal. México: Trillas.

Guzmán, S., \& Sánchez Escobedo, P. (2006). Efectos de un programa de capacitación de profesores en el 
desarrollo de habilidades de pensamiento crítico en estudiantes universitarios en el Sureste de México. Revista Electrónica de Investigación Educativa, 8(2), $1-17$.

Harry, J. M. (1984). A revised Measure of Approval Motivation and Its relationship to social desirability. Journal of personality assessment, 48(5), 508-519.

Havercamp, S. M., \& Reiss, S. (2003). A Comprehensive Assessment of Human Strivings: Test-Retest Reliability and Validity of the Reiss Profile. Journal of Personality Assessment, 81(2), 123-132.

Kellar, H., Treadwell, T. W., Kumar, V. K., \& Leach, E. S. (2002). The Personal Attitude Scale-II: A Revised Measure of Spontaneity. Journal of Group Psychotherapy, Psychodrama, \& Sociometry, 55 (1), 35-46.

Kerlinger, F. N., \& Howard, B. L. (2002). Investigación del Comportamiento. Métodos de Investigación en Ciencias Sociales (Cuarta ed.). México: McGraw-Hill.

Kolotkin, R. L., \& Crosby, R. D. (2002). Psychometric evaluation of the impact of weight on quality of lifelite questionnaire (IWQOL-Lite) in a community sample. Quality of Life Research, 11(2), 157-171.

Konstabel, K., Aavik, T., \& Allik, J. (2006). Social desirability and consensual validity of personality traits. European Journal of Personality, 20, 549-566.

Kumpikaite, V. (2007). Human resource training evaluation. Engineering Economics, 5(55), 29-36.

Loo, R., \& Thorpe, K. (2000). Confirmatory factor analyses of the full and short versions of the MarloweCrowne Social Desirability Scale. The Journal of Social Psychology, 140(5), 628-635.

Martínez Selva, J. (2005). La psicología de la Mentira. Barcelona: Paidos.

McGehee, W., \& Thayer, P. W. (1986). Capacitación: Adiestramiento y Formación Profesional. México: Limusa.

Mikolajczak, M., Luminet, O., Leroy, C., \& Roy, E. (2007). Psychometric properties of the Trait Emotional Intelligence Questionnaire: Factor structure, reliability, construct, and incremental validity in a french-speaking population. Journal of Personality Assessment, 88(3), 338-353.

Nunnally, J. C., \& Bernstein, I. J. (1995). Teoría Psicométrica (Tercera ed.). México: McGraw-Hill.
O'Grady, K. E. (1988). The Marlowe-Crowne and Edward Social Desirability Scale: A Psychometric Perspective. Multivariate Behavioral Research, 23, 87-101.

Paulhus, D. L. (1984). Two-component models of socially desirable responding. Journal of Personality and Social Psychology, 46, 598-609.

Paulhus, D. L., \& Reid, D. B. (1991). Enhancement and Denial in Socially Desirable Responding. Journal of Personality and Social Psychology, 60(2), 307-317.

Rahim, M. A. (1982). Reliability and validity of Likert's profile of organizational characteristics. Journal of Psychology, 112 (2), 153-157.

Rahim, M. A. (1983). Measurement of organizational conflict. Journal of General Psychology, 109 (2), 189199.

Rahim, M. A. (1988). The development of a leader power inventory. Multivariate Behavioral Research, 23(4), 491-503.

Ramanaiah, N. V., \& Martin, H. J. (1980). On the twodimensional nature of the Marlowe-Crowne Social Desirabilily Scale. Journal of Personality Assessment, 44(5), 505-514.

Ray, J. J. (1984). The reliability of short social desirability scale. The Journal of Social Psychology, 123, 133134.

Reynolds, W. M. (1982). Development of reliable and valid short forms of the Marlowe-Crowne Social Desirability Scale. Journal of Clinical Psychology, 38 (1), 119-125.

Robertson, K. R., \& Milner, J. S. (1983). Construct validity of the Child Abuse Potential Inventory. Journal of Clinical Psychology, 39(3), 426-429.

Rodríguez, M. (1993). Administración de la Capacitación. México: McGraw-Hill.

Salgado, J. F. (2005). Personalidad y deseabilidad social en contextos organizacionales: implicaciones para la práctica de la psicología del trabajo y las organizaciones. Papeles de Psicólogos, 26, 115-128.

Schludermann, S., \& Schludermann, E. (2007). A Revised Paternal Attitude Research Instrument (PARI) Q4: Methodological study. Journal of Psychology, 96(1), 15-23.

Seol, H. (2007). A Psychometric Investigation of the Mariowe-Crowne Social Desirability Scale Using Rasch Measurement. En: Measurement and Evaluation in Counseling cand Development, 40, 155-168. 
Smith, H. C., \& Wakeley, J. H. (1988). Psicología de la Conducta Industrial. México: McGraw-Hill.

Stöber, J. (2001). The Social Desirability Scale-17 (SDS17). European Journal of Psychological Assessment, 17(3), 222-232.

Stöber, J., Dette, D. E., \& Musch, J. (2002). Comparing continuous and dichotomous scoring of the Balanced Inventory of Desirable Responding. Journal of Personality Assessment, 78 (2), 370-389.

Strahan, R., \& Gerbasi, K. C. (1972). Short, homogeneous versions of the Marlowe-Crowne Social Desirability Scale. Journal of Clinical Psychology, 28(2), 191-193.

Van de Vijver, F. J. R. (1997). Bias research: Introduction. European Review of Applied Psychology, 47(4), 261262.
Van de Vijver, F. J. R. (1998). Towards a theory of bias and equivalence. ZUMA-Nachrichten Spezial, 3, 41-65.

Wichstrom, L. (1995). Harter's Self-Perception Profile for Adolescents: reliability, validity, and evaluation of the question format. Journal of Personality Assessment, 65(1), 100-116.

Williams, M. L., Podsakoff, P. M., Todor, W. D., Huber, V. L., Howell, J. P., \& Dorfman, P. W. (1988). A preliminary analysis of the construct validity of Kerr \& Jermier's Substitutes for Leadership Scales. Journal of Occupational Psychology, 61(4), 307-333.

Zook II, A., \& Sipps, G. J. (1985). Cross-validation of a short form of the Marlowe-Crowne Social Desirability Scale. Journal of Clinical Psychology, 41(2), 236-238. 